\title{
Arbor
}

\section{Pasado, presente y futuro de las bibliotecas universitarias españolas *}

\section{Lluís Anglada i de Ferrer y Margarita Taladriz Mas}

Arbor CLVII, 617-618 (Mayo-Junio 1997) 65-88 pp.

Los autores hacen un repaso de los diferentes elementos que han contribuido a que la imparable evolución de las Bibliotecas Universitarias haya sido posible. La propia evolución de las Instituciones de las que forman parte y el crecimiento económico de las diferentes áreas de investigación han favorecido dicho desarrollo. Se analizan la organización y los recursos humanos, la colección, los presupuestos y los edificios, el grado de automatización e implantación de nuevas tecnologías y los servicios que prestan a sus usuarios. Atención especial merece el apartado dedicado a la Cooperación a nivel nacional. Finalmente se avanzan cuáles son los retos y problemas a los que se enfrentan las Bibliotecas Universitarias de cara al 2000, cuya definición viene dada como resultado de una encuesta dirigida a todas ellas y de la que se concluye que la calidad y el liderazgo en la Institución son principales motivos de preocupación para los profesionales.

\section{La evolución de las bibliotecas universitarias españolas entre 1985 y 1995 (una década prodigiosa)}

Diversos autores hacen un diagnóstico muy parecido de la situación de las bibliotecas universitarias españolas en $1985^{1}$. Los principales problemas que se mencionan son:

* (Ponencia presentada a las IX Jornadas de Bibliotecarios Andaluces. Granada, Mayo, 1996) 


\section{Lluís Anglada i de Ferrer y Margarita Taladriz Mas}

- la falta de reconocimiento del papel de la biblioteca en la universidad $\mathrm{y}$

- la dispersión de fondos bibliográficos entre bibliotecas de facultad y departamentos,

- la desorganización general de los servicios bibliotecarios,

- la inadecuación y pobreza de recursos en general (fondos bibliográficos, locales, equipos, etc.),

- la falta de personal y pobre formación del personal existente,

- la falta de presupuestos para las bibliotecas universitarias.

Los autores no mencionan, en cambio, la falta de un buen nivel de automatización a pesar de que este tema será estelar a finales de la década y a principios de la siguiente.

Es un hecho significativo que el primer artículo publicado por el Boletín de la Anaba que menciona las bibliotecas de las universidades no aparece hasta $1970^{2}$ (y el segundo es de 1976). Mayor presencia empiezan a tener a partir de la década de los 80 pero, a pesar de ello, aspectos clave de la vida de las bibliotecas universitarias en estos años están sin documentar.

Hay un buen libro que nos permite conocer la situación de las bibliotecas universitarias del Reino Unido en la década de los $80^{3}$. Para redactarlo, su autor - Toby Burrows - analiza los datos e informaciones que le aportan las estadísticas de las asociaciones de bibliotecas universitarias (SCONUL y COPOL) así como los informes anuales que la mayoría de éstas vienen redactando desde hace muchos años. El estimulante libro de Thompson ${ }^{4}$ sobre la necesidad de un giro en la orientación de las bibliotecas universitarias se basa en las informaciones de las memorias anuales de las bibliotecas.

Las bibliotecas universitarias españolas no han seguido el ejemplo de sus colegas anglosajonas, por lo menos en lo que se refiere a la difusión anual de sus resultados y actividades. Las universidades Autónoma de Barcelona y de Zaragoza han sido las únicas que en los últimos años han editado y difundido memorias anuales. En 1994 este ejemplo ha sido seguido por otras universidades (Alcalá, Complutense, Las Palmas de Gran Canaria, Politécnica de Cataluña, Santiago ...) y es de esperar que empecemos a disponer de información continuada de las realizaciones, los problemas y los resultados de las bibliotecas universitarias españolas.

Hablar de las bibliotecas universitarias españolas es enfrentarse al problema de la falta de datos. Frente a las completas y continuadas secuencias estadísticas sobre bibliotecas universitarias de los países de nuestro entorno ${ }^{5}$, en España sólo existen tres estudios que incluyen 
datos completos de todas o de casi todas las bibliotecas universitarias. Estos son:

- un informe de 1983 elaborado por un grupo mixto de trabajo de los Ministerios de Educación y Cultura ${ }^{6}$,

- un informe de 1988 elaborado por Fundesco por encargo del Ministerio de Educación ${ }^{7}$,

- datos estadísticos completos de 1994 elaborados por el Consejo de Universidades y la Conferencia de Directores de Bibliotecas Universitarias y Científicas Española ${ }^{8}$.

Es sintomático que, de estos tres estudios fundamentales, sólo haya sido editado el citado en primer lugar. Estos informes incluyen datos muy relevantes sobre la situación de las bibliotecas universitarias, pero las metodologías usadas difieren y no siempre se tratan con igual intensidad. Por otra parte, existen otros tres buenos estudios, pero referidos solo a las bibliotecas universitarias de una parte del territorio español: el llamado territorio $\mathrm{MEC}^{9}$, Cataluña ${ }^{10}$ y las bibliotecas universitarias de la Comunidad Autónoma de Madrid ${ }^{11}$.

\section{El modelo, la organización y el personal}

Probablemente el grupo de bibliotecas españolas que haya avanzado más en los últimos diez años ha sido el de las bibliotecas universitarias. Los cambios han venido motivados por una reorganización de la universidad española que ha supuesto también la reorganización de sus bibliotecas.

La universidad española empieza a cambiar de forma notable con la Ley de reforma universitaria de 1983. Ésta configuraría un nuevo modelo para la universidad (democrática, científica y autónoma) que se define a partir del negativo de la universidad franquista (autoritaria, dirigida y centralista). En este contexto de redefinición, los bibliotecarios mostraron su preocupación por el lugar que ocuparía la biblioteca en el seno de unas universidades refundadas. La preocupación por la situación jurídica de la biblioteca, por que los estatutos obliguen a que ésta tenga una dirección técnica y por la falta de una política bibliotecaria a nivel estatal es una constante hasta bien entrada la década de los $90^{12}$.

Era lógico, las bibliotecas universitarias partían de una situación de total desgobierno y atomización ${ }^{13}$ que no parecía poder superarse más que con fuertes medidas organizativas expresadas en los propios estatutos de las universidades. Por otra parte, la reivindicación de 
tener un claro respaldo legal forma parte de la cultura profesional bibliotecaria que se siente más cómoda en situaciones perfectamente definidas y normativas que en situaciones fluidas y ambiguas.

Pero los grandes cambios de la década no han sido consecuencia de la mención o no de la biblioteca en los estatutos de la universidad o de la existencia o no de reglamentos de la biblioteca, sino más bien de la profunda reorganización de la universidad derivada de su autonomía y de las inyecciones económicas que se empiezan a recibir para la mejora de infraestructuras y servicios. Si bien, en este contexto, las primeras actuaciones de las universidades se dirigen a la investigación, pronto empiezan a orientarse a servicios "horizontales" como las bibliotecas.

Con las inversiones surgió la necesidad de que las universidades contaran con direcciones técnicas que se hicieran cargo de nuevos proyectos (por ejemplo, automatización), la planificación de nuevos recursos (edificios) y organizaran la gestión a partir de nuevas bases (centralización de la catalogación y de las adquisiciones). En muchos casos aparece una nueva hornada de directores de biblioteca a partir de escalas propias de la universidad (en substitución de los funcionarios del cuerpo estatal de Facultativos).

Los equipos directivos de las bibliotecas universitarias aprovecharon la situación y, además de encargarse de los nuevos proyectos, empezaron a organizar las bibliotecas de la universidad en un sistema bibliotecario coordinado que compartía políticas e instrumentos.

Como hemos mencionado, a partir de los procesos estatutarios que las universidades emprenden a partir de 1983, las bibliotecas intentan que los estatutos incluyan menciones a la biblioteca o que se aprueben reglamentos de la biblioteca. En ambos casos se postula una definición de la biblioteca como:

«... una unidad funcional en la que se organizan, procesan, custodian y ponen a disposición de los usuarios todos los fondos documentales de la Universidad, con independencia del soporte en que se hallen, del lugar en que estén depositados y de los fondos con cargo a los cuales hayan sido adquiridos. ${ }^{14}$,

Vale la pena observar que la definición propuesta insiste más en el aspecto patrimonial de la biblioteca que en el funcional. La obsesión en la segunda mitad de los años 80 fue el descontrol de los fondos bibliográficos dispersos (y por ello inaccesibles a los usuarios) entre bibliotecas de facultad y departamento en el mejor de los casos, y en 
Pasado, presente y futuro de las bibliotecas...

despachos particulares o desaparecidos, en el peor de ellos. Se tomó para copiar el modelo anglosajón que, altamente centralizado, era difícil de implantar en una universidad con un grado de masificación y de dispersión territorial como el de la española.

En la situación actual nos falta un modelo propio, un modelo que sin renunciar a imitar las virtudes (especialmente en servicios) de las ejemplares bibliotecas inglesas y norteamericanas, sepa también adaptarse a las especiales características de la universidad española. Sin duda alguna, las bibliotecas de área, campus o facultad siempre tendrán en nuestro caso más autonomía y especificidades que las de universidades con una biblioteca central y algunas «branch libraries». El quizá único modelo posible sea el que la literatura profesional viene llamando "semi-centralizado y coordinado". Excepto en casos contados ${ }^{15}$ la mejor forma de ver las bibliotecas de una universidad es como un sistema bibliotecario, con distintas unidades de prestación de servicios, con un mismo o parecido nivel, con políticas e instrumentos comunes y con más o menos autonomía de gestión con respecto a la dirección del sistema.

La organización de las bibliotecas universitarias ha supuesto el crecimiento de sus plantillas, la estabilización del personal y su estructuración. En $1980^{16}$ el personal de bibliotecas censado era de 416 (49 facultativos, 46 ayudantes y 321 auxiliares); en 1989, según el estudio de Fundesco, el total de personal era de 2.535 personas; y en 1994, un total de 3.419 (1.561 personal técnico y 1.858 personal auxiliar). La actual proporción entre personal técnico y auxiliar es del $45 \%$ frente al $55 \%$, una proporción de personal técnico notablemente superior al de las bibliotecas anglosajonas. Este hecho probablemente sea debido al mayor número de bibliotecas por universidad y a una mayor dedicación del personal técnico a tareas de catalogación en el caso español.

La historia debería documentar más aquellos no muy lejanos periodos en los que un número no despreciable de bibliotecarios eran pagados de la partida de fungibles o de personal de limpieza. La década de los 80 fue época de oposiciones y de estabilización del personal. Actualmente las bibliotecas universitarias españolas organizan a su personal en escalas de facultativos, de ayudantes, de auxiliares, de administrativos y de personal especializado.

La dirección, en la inmensa mayoría de casos, corresponde a personal técnico y las distintas bibliotecas y secciones están gestionadas por personal con complementos de jefatura. El periodo reseñado ha sido el de creación de servicios técnicos centrales que, típicamente, se han 
ocupado de la implementación de los sistemas de automatización y de la catalogación y adquisiciones centralizadas. Aunque los servicios técnicos no se hayan desarrollado en su plenitud en todas las universidades, sí ha sido al menos el modelo a seguir por parte de casi todas ellas. Actualmente se vive cierta tendencia a la descentralización de los servicios técnicos, esto posiblemente sea debido a la consolidación del sistema y a la pérdida de peso de la automatización y la catalogación.

Los cambios en el sistema de gestión de las bibliotecas universitarias y la estabilización de las plantillas se ven reflejados también en los planes de formación de personal. Si los bibliotecarios de principios de los 80 eran seleccionados de forma un tanto informal, los de finales de la década lo son después de rigurosas oposiciones y el periodo de autoformación del personal ha sido seguido por el de planes de formación organizados y mantenidos por la propia universidad y la dirección de la biblioteca. Las estadísticas de 1994 reseñan 331 cursos de biblioteconomía y 223 cursos generales con 2.081 personal técnico asistente a cursos y 778 asistentes entre el personal auxiliar.

\section{Fondos bibliográficos, presupuestos y edificios}

El análisis del estado de las bibliotecas universitarias en fondos, presupuestos y edificios ha de ser forzosamente, debido a los datos disponibles, cuantitativo. Las observaciones sobre la efectividad y eficiencia del uso de estos recursos solo pueden basarse en apreciaciones subjetivas y en conocimientos personales.

El número de volúmenes de monografías declarado en las estadísticas del Consejo de Universidades de 1994 era de 17.294.971. El mismo factor en el Informe que recogía datos de 1983 era de 6.089.070. En once años el incremento ha sido de 11.205.901 volúmenes de monografías (acercándose a un crecimiento del $100 \%$ ).

Estos números indican que la media de crecimiento anual ha sido de 1.018 .718 volúmenes/año. Esta cifra coincide bastante con los 852.994 volúmenes de nuevo ingreso del año $1994 \mathrm{y}$, en cualquier caso, indica un cambio de tendencia importante en el incremento de fondos con respecto a la situación acumulada hasta 1983.

Según los datos de 1994 , el $70,9 \%$ de las monografias de nuevo ingreso proceden de la compra y el $29,1 \%$ restante del canje y los donativos. Dando un precio aproximado de 4.000 ptas. a cada volumen (en pesetas de 1996), la inversión en la compra de monografias habría ascendido anualmente a unos 2.800 millones de pesetas, dato concordante también con los 3.133 declarados en las estadísticas de 1994. 
Pasado, presente y futuro de las bibliotecas...

$\mathrm{Si}$ analizamos el impacto de este crecimiento en volúmenes con respecto al número de usuarios potenciales, veremos que la ratio libros por usuario eran 10,85 en 1983, 9,50 en el territorio MEC en 1987, 13,51 en 1988, 15,51 en las bibliotecas catalanas en 1992 y de 11,86 en 1994. Los crecimientos en estos últimos años han sido una carrera para atrapar nuestra propia sombra. Los incrementos en fondos bibliográficos han corrido paralelos al aumento de población universitaria y el crecimiento de casi el $100 \%$ en volúmenes totales se corresponde a un incremento de menos de un $10 \%$ en la ratio volumen/usuario.

Dejando al margen la comparación de la situación española con la de países de nuestro entorno referencial, las conclusiones podrían ser optimistas. Pero deberíamos analizar además dos factores: la calidad de estos fondos y su uso. Poco podemos decir al respecto, pero valga una muestra como dato significativo: un estudio de 1990 hecho en la Universidad Politécnica de Cataluña indicaba que solo un $60 \%$ de la bibliografia recomendada en las guías docentes estaba presente en las bibliotecas de la universidad.

Según las estadísticas de 1994, las universidades españolas destinaron 3.133.453.343 ptas. a la compra de monografias (un 45,72\% del presupuesto de adquisiciones), 3.450 .238 .906 ptas. a las subscripciones de revistas (un 50,34\%) y 270.588 .004 ptas. a la adquisición de otros fondos bibliográficos (un $3,94 \%$ ).

A pesar de la existencia de algunos estudios específicos ${ }^{17}$, los datos estadísticos disponibles no indican de forma clara la procedencia de estos presupuestos. Alrededor de un $30 \%$ parece proceder de los departamentos, pero el tema de fondo es saber si los materiales bibliográficos adquiridos con estas partidas están depositados en alguna biblioteca y son accesibles por los usuarios. De datos de $1994^{18}$ se desprende que un $90,48 \%$ de las bibliotecas de las universidades conforman las facturas de las compras realizadas por los departamentos, que en un $56,10 \%$ de universidades los departamentos hacen compras directas y que un $46,86 \%$ de universidades tienen centralizada la compra de materiales bibliográficos. Estos datos son poco indicativos de la realidad. Si bien unas pocas bibliotecas logran garantizar el control de todos los fondos bibliográficos y garantizan su accesibilidad pública, en la mayoría de ellas una parte importante de dinero de procedencia pública sirve para comprar fondos bibliográficos que no pueden ser utilizados por toda la comunidad universitaria.

Un problema adicional que tampoco está suficientemente documentado es el de la consolidación de las partidas económicas destinadas a la compra de materiales bibliográficos. Como se ha mencionado, la 


\section{Lluís Anglada i de Ferrer y Margarita Taladriz Mas}

universidad española ha vivido unos años de bonanza económica. En este contexto ha sido fácil que determinados excedentes a fin de ejercicio revirtieran a bibliotecas. Algunos de los presupuestos conocidos de bibliotecas universitarias han estado nutridos de forma importante por partidas extraordinarias y, por tanto, no consolidadas. Los próximos años de restricción económica hacen temer que los actuales niveles de financiación de compras no puedan mantenerse.

En el capítulo de edificios, los metros cuadrados construidos dedicados a bibliotecas eran 229.093 en 1988 según el estudio de Fundesco y 422.801 en 1994. El incremento ha sido de $193.708 \mathrm{~m}^{2}$ en seis años, a una media de $32.284 \mathrm{~m}^{2}$ añadidos por año. Puestos a seguir especulando, podríamos decir que todas las universidades españolas han hecho una nueva biblioteca de unos $700 \mathrm{~m}^{2}$ cada año. Valorando a precios de 1995 en 135.000 ptas el metro cuadrado construido y equipado, la inversión en edificios y mobiliario ha sido de 26.150 millones de pesetas en seis años, a una media de 4.358 millones de pesetas por año.

La traducción de estas cifras a ratio $\mathrm{m}^{2} /$ usuario es la siguiente: $0,20 \mathrm{~m}^{2}$ /usuario en el territorio MEC en 1987, 0,23 en 1988, 0,31 en las bibliotecas catalanas en $1992 \mathrm{y}$, finalmente, 0,30 en las estadísticas de 1994. En este caso, como en el anterior de fondos, al crecimiento en valores absolutos de cerca de un $100 \%$ en $\mathrm{m}^{2}$ construidos para biblioteca del periodo 1988-1994, le corresponde un crecimiento en valores relativos inferior al $50 \%$ y los grandes esfuerzos de inversión en ladrillo ven disminuido su impacto por el crecimiento de la población universitaria en los últimos años.

Las cifras engañan. El salto, aunque esté poco documentado, ha sido cualitativo. En los últimos diez años se han construido bibliotecas nuevas, modernas en su concepción y organización y de grandes dimensiones (en comparación con la situación anterior). Debería hacerse una censo de las bibliotecas universitarias con más de $5.000 \mathrm{~m}^{2}$. Actualmente cuentan con bibliotecas de estas dimensiones al menos las universidades: Autónoma de Barcelona, de Barcelona, de Cantabria, Carlos III, de Castilla-la Mancha, Complutense, de La Laguna, UNED, y Pública de Navarra y están construyendo bibliotecas de estas características la de Alicante, la Autónoma de Madrid, las politécnicas de Cataluña y de Valencia, la Pompeu Fabra ...

\section{Automatización y nuevas tecnologías}

El proceso de automatización de las bibliotecas de las universidades españolas es sin duda el aspecto más documentado de la vida de éstas. 
Significativamente, de las seis publicaciones a tener en cuenta ${ }^{19}$, tres están escritas en inglés y publicadas fuera de España.

Entre 1980 y 1995 se vive en España, de forma comprimida, la historia de la automatización de las bibliotecas que, de forma más dilatada, se viene desarrollando a nivel mundial desde los lejanos años 60. En 1980 se desarrolla el primer sistema «in house» de automatización de bibliotecas entre las Universidades Politécnica de Barcelona y la de Barcelona y la Red de bibliotecas de "La Caixa». A estos desarrollos les siguieron otros: los MDUP Y MBUP de la Universidad Politécnica de Cataluña, el BUBIS de la Universidad de Barcelona y el de la Universidad Politécnica de Valencia.

A principios de la década de los 80 se instala el primer sistema comercial de automatización de bibliotecas en una universidad: el DOBIS/LIBIS en la Universidad de Navarra y, algo más tarde, el sistema comercial SABINI se implementa en algunas universidades (Cantabria). A pesar de ello, a finales de los 80 la automatización de las bibliotecas no había despegado aún. El motivo se encontraba en que los desarrollos "in house" eran demasiado caros de mantener y, por otra parte, la oferta de sistemas comerciales era aún demasiado escasa. A principios de los años 90 la situación cambia radicalmente con la comercialización en España de nuevos sistemas.

A pesar de los esfuerzos comerciales de GEAC, este sistema no encuentra compradores, pero sí los sistemas VTLS (de origen norteamericano) y LIBERTAS (de origen inglés). La aparición de estos productos vigoriza el mercado y produce un vuelco en la situación. En mayo de $1995^{20}$ un $89,36 \%$ de las bibliotecas universitarias españolas indican que sus catálogos están automatizados. La distribución entre sistemas era, a principios de 1985 , la siguiente:

$\begin{array}{lrr}\text { - Absys } & 2 & 2 \% \\ \text { - Biblio 3000 } & 1 & 2,5 \% \\ \text { - Coral } & 1 & 2,5 \% \\ \text { - DOBIS/LIBIS } & 12 & 30 \% \\ \text { - LIBERTAS } & 10 & 25 \% \\ \text { - Sabini } & & 10 \% \\ \text { - VTLS } & & 25 \%\end{array}$

Los procesos de automatización se han iniciado típicamente por la catalogación ${ }^{21}$. A medida que las bibliotecas iban automatizando la catalogación se preocupaban también de la retroconversión de las 
fichas manuales ${ }^{22}$. La evolución de los registros bibliográficos automatizados da un salto en los últimos años. Los 2.027.260 de 1992 citados por Ortiz-Repiso/Ríos (correspondientes al 14,40\% del total de los fondos) se transforman en 5.796 .876 a finales de 1994 (correspondientes a un $34,78 \%$ del total) ${ }^{23}$.

Al mismo tiempo, han cobrado un gran impulso los catálogos colectivos automatizados. A nivel mundial, el gran momento histórico de creación de catálogos cooperativos fueron los años 70 e inicios de los 80 , y son justamente éstos los que facilitan las automatizaciones locales. En España en cambio, éstos no aparecen con fuerza hasta que la base de automatización a nivel local es lo suficientemente amplia y se desarrollan alrededor de las bibliotecas usuarias de un sistema. Cabe destacar la Red Universitaria Española DOBIS/LIBIS (1990) con un catálogo colectivo de casi un millón de registros, el Catálogo Colectivo de las Universidades de Cataluña (1995) con un millón y medio de volúmenes y la Base de datos bibliográfica SLS España. La asociación Rebiun inició en 1992 un CD-ROM con los registros bibliográficos de algunas de las bibliotecas miembros y contaba a finales de 1994 con 941.813 registros en su CD-ROM.

La teledocumentación apareció pronto en las universidades (a veces en organizaciones al margen de la biblioteca) pero ésta no ha sido una actividad que se haya desarrollado de forma espectacular. Las cifras de consultas a servicios de teledocumentación de las distintas universidades (21.658 en 1994) no parecen ser muy significativas frente a las realizadas a bases de datos en CD-ROM (183.552 en 1994). Este fenómeno, comparable a lo acaecido en otros países, puede verse reforzado en nuestro caso por la tardía aparición de servicios de teledocumentación, aparición que es casi paralela a los primeros CD-ROM.

Las bases de datos en CD-ROM se instalan rápidamente en las universidades españolas y muy pronto se implementan redes de CDROM (la primera fue la de la Universidad Politécnica de Cataluña en 1991). Según los datos estadísticos de 1994, de las 46 universidades encuestadas, 43 manifiestan tener bases de datos en CD-ROM (un total de 2.479 bases de datos y un promedio de 58 títulos por universidad) y 30 declaran tener algunas instaladas en red.

Las nuevas tecnologías encuentran en las universidades un campo abonado para florecer y las bibliotecas saben aprovechar su situación. Algunas bibliotecas universitarias han usado hojeadores de Internet para informar de sus servicios. La Universidad Jaume I fue la pionera y sus desarrollos de información sobre Gopher aplicado a sus bibliotecas fueron seguidos con mucho interés e imitadas por otras bibliotecas 
Pasado, presente y futuro de las bibliotecas...

(U. Cantabria). Actualmente la tendencia de las bibliotecas es mantener información en las páginas Web de la Universidad (U. Autónoma de Barcelona, U. Complutense, por ejemplo) o mantener servidores Web de las propias bibliotecas (Universidad Politécnica de Cataluña).

Los desarrollos tecnológicos de las bibliotecas universitarias españolas han sido espectaculares especialmente en los últimos cinco años. Nuestra situación resiste bien la comparación con la de países más avanzados. En comparación con éstos, nos acercamos a su nivel en automatización local. Bien es cierto que estamos aún lejos de las facilidades proporcionadas por servicios nacionales como el catálogo nacional de revistas de Francia o el servicio BIDS en el Reino Unido.

El momento presente es complejo desde el punto de vista tecnológico. Algunos desarrollos pueden poner en cuestión actividades tradicionales de las bibliotecas desde principios de siglo. A pesar de ello, se debe tener en cuenta que las opciones de futuro van a estar configuradas no sólo por los nuevos desarrollos tecnológicos sino por el hecho de que éstos encuentren viabilidad económica y mejoren las prestaciones de los sistemas actuales ${ }^{24}$.

Las bibliotecas universitarias deberán prestar especial atención en los próximos años a los desarrollos tecnológicos en las áreas siguientes:

- bases de datos instaladas localmente,

- CD-ROM en red cooperativa entre diversas universidades,

- cooperativas de redistribución de bases de datos,

- acceso a bases de datos en línea con licencia de campus,

- sumarios de revistas en formato electrónico,

- organización de recursos electrónicos en Internet,

- acceso electrónico a los documentos,

- archivo de los documentos electrónicos de la universidad.

La enorme movilidad de la oferta de servicios comerciales en el dominịo de la información electrónica, las grandes posibilidades de las nuevas tecnologías y los nuevos costes asociados a ellos no permitirá que las bibliotecas universitarias se mantengan al día de forma fácil.

\section{Los servicios}

Aunque el nivel de los servicios prestados por las bibliotecas ha aumentado en los últimos años de forma significativa, éste es el apartado más difícil de documentar con cifras. Es indudable que los servicios y equipamientos bibliotecarios se han ampliado y modernizado. Lo dicho 


\section{Lluís Anglada i de Ferrer y Margarita Taladriz Mas}

hasta ahora sobre la organización de las bibliotecas, sus fondos y recursos y la automatización así lo confirman.

Las bibliotecas se han equipado con herramientas que han permitido mejorar los servicios ofrecidos: terminales de OPAC (un promedio de 24 por universidad), Pcs y terminales para la gestión y la administración (32 y 28, respectivamente, por universidad), Pcs conectados a redes de CD-ROM (13 por universidad), lectores monopuesto de Cds (14 de media), lectores de microformas ( 24 de media), reproductoras de vídeos, casetes, discos compactos y videodiscos $(21,39,4$ y 2 , respectivamente, de media por universidad), fotocopiadoras en régimen de autoservicio (15 de media), faxes (5 por universidad) y equipos de seguridad antihurto.

Los horarios de apertura de las bibliotecas se han ampliado. La media es de 63 horas por semana (bastante superior a la media de las bibliotecas universitarias francesas). El dato es poco significativo por ser justamente una media que esconde los numerosos casos de bibliotecas abiertas en días festivos y en turnos de noche (por ejemplo en las bibliotecas de las universidades de Alcalá de Henares, Autónoma de Barcelona, de Barcelona, Politécnica de Cataluña, Pompeu Fabra, Rovira i Virgili, de Cantábria, etc.)

Es difícil evaluar la calidad de las instalaciones que es muy diversa. También lo es cuantificar y evaluar el uso de sus instalaciones. Recientemente algunas universidades (Complutense y Politécnica de Cataluña) han realizado encuestas entre sus usuarios para conocer el uso que los clientes hacen de la biblioteca, pero no se pueden generalizar los datos. Las conclusiones de la encuesta de la UPC ${ }^{26}$ destacan:

- que un 29,46\% de los estudiantes visitan la biblioteca diariamente, un $43,77 \%$ entre 2 y 3 veces por semana y un 13,32 $\%$ una vez por semana,

- que el motivo de la visita el día de la encuesta fue, para un $39,50 \%$ de los estudiantes, la consulta de material propio, para un $7,48 \%$, la consulta de material de la biblioteca, para un $25,59 \%$ la consulta de material propio y de la biblioteca, y, para un $21,43 \%$ la búsqueda de información,

- que un 27,57 \% de los estudiantes encuestados pasa menos de una hora en la biblioteca, mientras que el $46,10 \%$ pasa entre una y tres horas y un $26,33 \%$ pasa más de tres horas en su visita,

- que los principales motivos de insatisfacción el día de la encuesta fueron derivados de plazas insuficientes, de mala climatización y del ruido ambiental. 
Pasado, presente y futuro de las bibliotecas...

Sin duda alguna las bibliotecas son muy utilizadas. Lo son lo suficiente como para que casi todas las universidades conozcan los múltiples métodos usados por los estudiantes para conseguir reserva de plaza de lectura en las bibliotecas (había incluso quien montó un pequeño negocio con ello).

El uso mayoritario de las bibliotecas como salas de estudio ha sido profundamente debatido entre los profesionales del sector. El tema es ciertamente complejo. Por una parte, la función de la biblioteca universitaria también es la de ofrecer un entorno que facilite el estudio y fomente la curiosidad científica; por otra, la masiva afluencia de usuarios que tienen como único objetivo memorizar apuntes aleja de la biblioteca a aquellos que usarían sus fondos y servicios.

El problema quizá sea doble. En primer lugar, las ratios de espacio por miembro de la comunidad universitaria son muy bajos en relación con los de otros países. La mejora en este aspecto disminuiría la masificación y la falta de espacio. En segundo lugar, el problema del estudio en las bibliotecas no depende de las condiciones de éstas, sino de las exigencias curriculares que se orientan masivamente hacia la memorización. Pero la evolución obligada y, con los nuevos planes de estudio, ya perceptible, de la enseñanza universitaria, nos obliga a estar preparados para un contexto distinto en el que se va a requerir más espacio de biblioteca para el trabajo intelectual y (también) para el estudio.

Tenemos aún menos datos sobre el uso de estos fondos. La ratio anual de préstamos por usuario era de 5,82 en las bibliotecas catalanas en 1992 y de 4,71 en las estadísticas de 1994. Buscando los mejores resultados locales, veríamos ratios que se aproximan a los 10 préstamos por usuario/año pero que no consiguen superar esta magnitud. Como referencia podríamos citar que los ratios de bibliotecas universitarias inglesas se aproximan a los 30 préstamos por usuario/año y que los de las bibliotecas norteamericanas superan aún esta cifra.

El uso (comparativamente) bajo de nuestros fondos probablemente sea debido a una combinación de factores: poca accesibilidad de los fondos (dispersión de fondos en departamentos, una parte importante de libros en acceso cerrado), poca calidad de los fondos, pocas facilidades en el préstamo ... pero seguramente indica también que el método docente de la universidad española está basado en la memorización de los apuntes de clase y que no se exige o fomenta la consulta de material bibliográfico para los trabajos de curso.

Ciertamente, uno de los puntos a mejorar en nuestras bibliotecas universitarias sea el del acceso libre a los documentos. La base de 
la biblioteconomía anglosajona se asienta en este pilar, pero las bibliotecas de las universidades de la Europa continental han imitado tarde este ejemplo. Las bibliotecas de una universidad con unos servicios bibliotecarios de muy buen nivel como los de la Universidad Autónoma de Barcelona no empiezan a poner sus fondos en acceso libre hasta el 1986. Y la fecha de fundación de la universidad (1969) no permite aducir excusas derivadas del lastre de la historia. Conocemos los datos de documentos en acceso cerrado de la Universidad Complutense (un $72,27 \%$ de sus fondos en 1994) que, sin ser extrapolables a la media nacional, sin duda son indicativos de una situación que debe aún mejorar mucho.

Quizá el segundo gran reto en servicios sea el de la información bibliográfica y la atención al cliente. Por distintos motivos en parte históricos de formación de las bibliotecas y en parte estructurales de la universidad española, nuestras bibliotecas no se distinguen por ser tenidas por la comunidad universitaria como fuente de solución a sus problemas de información. La distribución de tareas entre el personal, según las estadísticas de 1994, muestran que sólo el 21,30 \% del tiempo del personal técnico se dedica a tareas de atención al público (frente a un $62,82 \%$ del personal auxiliar). La catalogación sigue consumiendo recursos profesionales importantísimos (un 37,17 \%), seguidos por la gestión (18 \%), las adquisiciones $(14,64 \%)$ y el préstamo interbibliotecario $(7,16 \%)$.

El reto de la mejora de la atención al público y la información bibliográfica es la asignatura pendiente de las bibliotecas universitarias y éste pasa no sólo por la reorganización de las bibliotecas y de su personal sino por un cambio de mentalidad de éste. Los puntos de servicios de las bibliotecas desatendidos por personal técnico y los bibliotecarios encerrados en salas de trabajo es una característica demasiado frecuente. Podemos ser más optimistas respecto la formación de usuarios. Esta tarea ha pasado a ser una actividad cotidiana en muchas bibliotecas.

\section{La cooperación}

España no es un país muy rico en experiencias de cooperación bibliotecaria aunque es entre las universidades donde han tenido mayor lugar. Para analizar correctamente esta afirmación se han de tener en cuenta al menos dos factores. 
Pasado, presente y futuro de las bibliotecas...

En primer lugar, hay dos formas de cooperación: la que nace de bibliotecas individuales que constituyen organismos superiores (esta modalidad se ha dado típicamente en países anglosajones, y la que se fomenta o crea desde organismos de la administración central (por ejemplo, los casos franceses o de países nórdicos). La modalidad que, por motivos culturales y de proximidad, sería más cercana al caso español sería la segunda, pero en España los esfuerzos cooperativos han surgido de las bibliotecas y han estado escasamente apoyados por la administración.

En segundo lugar, debemos recordar que hasta bien entrada la década de los ochenta las bibliotecas de una universidad son realidades totalmente disgregadas, sin funcionar como sistema. La cooperación no puede iniciarse sin una mentalidad y tradición cooperativas previas y éstas se adquieren a fuerza de trabajar conjuntamente. Cooperar es compartir, pero también es consensuar, ceder, adaptarse, a veces, perder autonomía... Hace poco más de diez años que los profesionales de las universidades han empezado a relacionarse entre sí y a trabajar conjuntamente, a pesar de pertenecer a una misma institución, estar sólo a cientos de metros de distancia y realizar las mismas tareas.

A pesar de su interés, no vamos a entrar aquí en las distinciones entre la terminología de la cooperación ${ }^{27}$. A efectos prácticos vamos a distinguir entre asociaciones, catálogos colectivos en línea, y organismos de coordinación.

En 1988 nace Rebiun (REd de BIbliotecas UNiversitarias) con nueve bibliotecas universitarias miembros. Actualmente participan 17. Rebiun ha sido una de las asociaciones más activas, sus actividades cooperativas se han dirigido a la formación, a la mejora del préstamo interbibliotecario y a la edición de un CD-ROM con los registros bibliográficos de las bibliotecas participantes.

$\mathrm{Al}$ mismo tiempo surgen asociaciones de bibliotecas especializadas. La más antigua es la Coordinadora de Documentació Biomèdica, fundada en 1983 y formada por bibliotecas de hospitales, facultades de medicina, farmacia, veterinaria y química y organizaciones médicas. La Coordinadora está implantada sobre todo en Cataluña y tiene por finalidad 'promover la cooperación y la racionalización de los recursos documentales en el ámbito de la biomedicina' y mantiene un catálogo colectivo de revistas biomédicas ${ }^{28}$.

Documat agrupa las bibliotecas de las facultades de matemáticas de las principales universidades españolas. Inició sus actividades en 1988 y mantiene un catálogo colectivo de revistas a partir del cual organiza planes cooperativos de compras. La $A B B A$ (Asociación de Bi- 
bliotecarios y Bibliotecas de Arquitectura) está formada por bibliotecas y bibliotecarios de escuelas y asociaciones profesionales de arquitectura e inició sus actividades en 1991. MECANO (heMEroteCA virtual área tecNOlogía) es la asociación especializada más reciente y está formada por bibliotecas de escuelas de ingeniería.

La progresiva automatización de las bibliotecas de las universidades ha supuesto también la creación de catálogos colectivos en línea. Las bibliotecas han constituido grupos de usuarios de los sistemas DOBIS-LIBIS, VTLS y Libertas a partir de los cuales se han iniciado algunos proyectos cooperativos. Doce universidades usuarias del sistema DOBIS/LIBIS forman en 1990 Ruedo (Red Universitaria Española DO$B I S / L I B I S)$ que mantiene un catálogo colectivo en línea ${ }^{29}$. Algunas universidades usuarias del sistema Libertas producen y mantienen de forma cooperativa la Base de datos bibliográfica SLS España a efectos de catalogación por copia y de facilitar la retroconversión de registros bibliográficos manuales.

Los cambios en la organización universitaria que supusieron la dependencia de las universidades de las comunidades autónomas aumenta la cooperación en base territorial. Las bibliotecas universitarias de Cataluña, que ya habían iniciado una cooperación a principios de los ochenta con un catálogo colectivo de revistas, están en proceso de creación del Consorci de Biblioteques Universitaries de Catalunya. El proyecto empezó en 1991 y actualmente funciona un catálogo colectivo en línea entre todas las universidades.

En agosto de 1993, en el marco del Congreso de la IFLA en Barcelona, se mantienen contactos informales entre bibliotecas de diferentes universidades y el 24 de noviembre del mismo año se constituye en Barcelona la Conferencia de directores de bibliotecas universitarias $y$ científicas españolas (CODIBUCE). La convocatoria partía de la necesidad de tener un ámbito de discusión y formulación de propuestas para aumentar la coordinación y la cooperación entre las bibliotecas, de la misma manera que existían organizaciones similares en Europa.

La Conferencia parte de la base de que, a pesar de que las situaciones en las bibliotecas pueden ser diversas, muchos problemas son comunes y algunas soluciones sólo serán posibles si se buscan conjuntamente. Los objetivos de la Conferencia son:

- ser un órgano de discusión, análisis, intercambio de información y de prospectiva,

- proponer actuaciones cooperativas o de coordinación que supongan un beneficio para los usuarios de las bibliotecas universitarias y científicas españolas, 
Pasado, presente y futuro de las bibliotecas...

- ser un organismo en el que estén representadas todas las bibliotecas universitarias, y que, como tal, pueda servir de interlocutor con la administración,

- contribuir a elevar el nivel de servicios y de recursos de las bibliotecas a través del intercambio de información, de la formulación de propuestas y del diálogo con la administración.

La Conferencia ha iniciado actuaciones cooperativas en los ámbitos de: estadísticas de bibliotecas, préstamo interbibliotecario, interconexión de sistemas automatizados, difusión de información sobre bibliotecas universitarias y normas para bibliotecas universitarias.

La finalidad y objetivos del ámbito de estadísticas es mejorar los instrumentos de gestión y planificación a partir del conocimiento de la situación actual, comparar los datos actuales con anteriores para determinar la evolución de los servicios bibliotecarios en los últimos años y tener las bases para formular normas adaptadas a la situación española. La Conferencia propuso un formulario estadístico al Consejo de Universidades y este organismo recogió los datos de 1994 que serán publicados en 1995.

En préstamo interbibliotecario, los objetivos son establecer las condiciones que permitan homogeneizar estos servicios para facilitar su uso, promover mejoras (reducción de costes de gestión y de tiempo de respuesta, simplificación de la administración ...). Las actividades desarrolladas han sido la aprobación de un acuerdo para todas las bibliotecas universitarias, la elaboración de un directorio de centros de préstamo accesible por Internet, el fomento del correo electrónico para la tramitación de peticiones y una propuesta de unificación de tarifas.

Bajo el epígrafe de Interconexión y cooperación, la Conferencia ha elaborado un programa de actuaciones dirigidas a incrementar el uso de RedIris en las bibliotecas, la mejora del acceso a los sistemas automatizados de bibliotecas, la creación de un catálogo colectivo en CD-ROM de bibliotecas universitarias, la aplicación de tecnologías de información a los servicios bibliotecarios, conseguir ayudas para la compra de bibliografia, equipos e infraestructura e incorporar los fondos bibliográficos procedentes de proyectos de investigación a las bibliotecas.

Más recientemente la Conferencia ha iniciado dos líneas de trabajo nuevas. La primera es establecer dos centros (las escuelas de biblioteconomía de Barcelona y de Granada) que recojan y difundan información y documentación sobre las bibliotecas universitarias. La segunda es iniciar los estudios y las discusiones para establecer unas normas para bibliotecas universitarias que se ajusten a las necesidades del país. 


\section{Los principales retos y problemas de las bibliotecas universitarias}

Con objeto de poder llegar a tener una percepción real de la situación a la que se enfrentan las Bibliotecas Universitarias en estos momentos, se diseño un cuestionario sencillo en el que se recogían algunos retos ante el futuro y problemas que afectan a las Bibliotecas Universitarias. Se pedía una valoración, según su importancia para las Bibliotecas encuestadas, de los diferentes retos y problemas. Se incluían además la posibilidad de añadir, de forma abierta, todas aquellas sugerencias que se consideraban de interés.

Hubo una respuesta importante ya que se enviaron 52 cuestionarios y se recibieron 35 respuestas, un $68 \%$. Se incluían todas las Universidades públicas y algunas privadas que habían participado en la última Conferencia de Directores y que estaban incluidas en el directorio que actualizó dicha Conferencia en Septiembre de 1995.

Del análisis global de las respuestas se deduce un hecho muy significativo: en general se concede mucha más importancia a los retos que a los problemas. Tendríamos que aclarar que se entienden por retos aquellos objetivos que nos vienen dados por el mundo que nos rodea: inserción en la institución, nuevos modelos de gestión, las nuevas tecnologías, etc.; mientras que los problemas se refieren más a lo cotidiano, al día a día, y, por ser más inmediatos, posiblemente se consideren más fáciles de afrontar.

Quizá lo más significativo de las respuestas sea la unanimidad respecto de lo que se considera el reto más importante. Un $90 \%$ de los encuestados respondieron que el crear servicios de calidad para un público cada vez más exigente y más autómo. Esta respuesta denota una clara vocación de servicio, un ser conscientes de que hay que estar al tanto de lo que el cliente espera de nosotros. De todos es bien sabido que "el nivel de calidad no lo pone el que presta el servicio sino aquel que lo recibe» ${ }^{30}$. Por vez primera, de forma casi unánime, los gestores de bibliotecas perciben que los usuarios y su satisfacción es su principal razón de ser. Es éste un cambio importante en la concepción que tradicionalmente se ha venido teniendo de las bibliotecas si bien es verdad que las universitarias siempre han tenido bastante claro que la razón de su existencia se basa en "el apoyo que prestan a la docencia y la investigación" ${ }^{31}$.

Solamente en un caso no se considera este reto como de importancia máxima, se trata de una biblioteca de nueva creación que opina que ya cuando pone en marcha nuevos servicios lo hace con criterios de 
Pasado, presente y futuro de las bibliotecas...

CALIDAD, por ello no tienen que llevar a cabo planes especiales para mejorar los servicios actuales.

En segundo lugar y con un $75,5 \%$ de bibliotecas que le conceden una importancia máxima se sitúa el reto de crear una organización adaptable a los cambios y abierta a la reorganización de los servicios. Las bibliotecas que más importancia le conceden son las bibliotecas con mayor tradición y, por ello, con un mayor lastre de anquilosamiento en su estructura funcional. Son aquellas bibliotecas que tienen una mayor dificultad para la movilidad del personal y para el cambio de funciones.

La posibilidad de aplicación de nuevas tecnologías a los servicios es muy importante para un $58 \%$ de las bibliotecas encuestadas, lo que demuestra que existe una clara conciencia del avance tecnológico, y de cómo afecta a los Servicios de Información. Un $23 \%$ le concede una relativa importancia a este hecho, no se sabe si porque inevitablemente la fuerza de los hechos les arrastrará a aplicarlas o porque se consideran suficientemente preparadas y apoyadas para poder afrontarlo. Un par de bibliotecas hacen constar el retraso tecnológico, esto es: la lentitud en la integración de las innovaciones tecnológicas.

Hasta hace sólo 5 años la automatización de servicios y fondos era uno de los retos fundamentales a afrontar. Hoy sólo un $45 \%$ de las bibliotecas le conceden una importancia relativa. Este $45 \%$ está formado, en su mayoría, por bibliotecas de nueva creación que han podido poner en marcha programas de automatización desde el principio. Para universidades de tradición como la Complutense, la Central de Barcelona, Salamanca, Granada, Zaragoza u Oviedo, este reto es de gran importancia, fundamentalmente por toda la carga retrospectiva de revistas y por lo que supone de tareas de coordinación en estructuras normalmente descentralizadas.

La Cooperación bibliotecaria y la política estatal de biblitecas tiene una importancia relativa, sólo un $40 \%$ le conceden una importancia alta/media. Se considera un reto importante pero no prioritario, consecuencia posiblemente de dos puntos.

- Un afán de cooperación a título individual/institucional resuelto de forma voluntarista.

- Una conciencia más o menos clara de que es inabordable una política estatal como consecuencia de las transferencias en materia de Educación de la Autonomía Universitaria y de la diversidad de políticas bibliotecarias en función de las diferentes autonomías. Mientras que en Cataluña se ha constituido un consorcio que integra Universitarias y la Biblioteca de Cataluña, 
en otras autonomías se continúa con un cierto afán individualista y sin un interlocutor válido, en la mayoría de las ocasiones, que sea capaz de aglutinar y dinamizar iniciativas válidas.

Curiosamente la convergencia de Servicios Informáticos y de Información con el Servicio de Biblioteca que es una tendencia clara en el mundo anglosajón con la creación del C.I.O. (Chief Information Officer), persona que coordina y dirige todo lo relacionado con el mundo de la Información, tanto lo que se refiere a recursos como a herramientas, no se considera importante en nuestro ámbito. Sólo un $33 \%$ le concede una gran importancia, mientras que un $32 \%$ le conceden una importancia mínima y en 2 ocasiones se obvia la respuesta. Entre los que le conceden una gran importancia se sitúan las universidades de nueva creación con excepción de Salamanca, que quizá se hallan inmersas en una dependencia funcional y orgánica diferente de las de más tradición y en las que existe un mayor acercamiento a los Servicios Informáticos.

Respecto de los problemas apuntados, al que se le concede una mayor importancia es al del liderazgo: Esto es, el tratar de conseguir una posición central en las actividades de la Universidad. Seis Universidades le conceden una importancia mínima, no es posible deducir de las respuesta si es porque ya ocupan esa posición central y no es, pues, un problema para ellas o es porque no conceden importancia a la posición que ocupen en la Institución. Son: Carlos III, Coruña, Rovira Virgili, Politécnica de Valencia, U. Pública Navarra y Granada. Un $53 \%$ que incluye las Universidades de más tradición: Barcelona, Complutense, Deusto, Salamanca, Oviedo, lo consideran un problema muy importante.

Una Biblioteca apunta que más que liderazgo hablaríamos de imagen... En realidad se le quería dar el sentido, en el cuestionario remitido, de si se contaba con la biblioteca en la Institución como algo importante capaz de participar de la vida universitaria a diferentes niveles: planes de estudio, actividades en general, capacidad de representación etc., etc.

En segundo lugar se sitúan de forma paralela 2 problemas: $L a$ formación y motivación del personal y la inadecuación e insuficiencia de locales $e$ instalaciones. En el primer caso este problema afecta de forma indiscriminada a todo tipo de bibliotecas, de nueva creación o tradicionales. En este apartado y dentro del capítulo observaciones se insiste en que por lo que respecta a temas de RR.HH., la inadecuación de las plantillas y la no participación en la elaboración de RPT, hace que los RR.HH. no se adapten a las necesidades reales y cada día más cambiantes de las Bibliotecas Universitarias, ello unido a una 
Pasado, presente y futuro de las bibliotecas...

cierta falta de profesionalidad de las plantillas actuales, sobre todo cuando se parte de un personal reclasificado y no de personal profesional en los diferentes niveles F.P., Diplomatura, etc.

Respecto de las carencias en infraestructura es, como en el caso anterior, sentido por bibliotecas de todo tipo. En las de nueva creación porque, con frecuencia, se crea el servicio sin cumplir unos mínimos imprescindibles y en las de más tradición porque las instalaciones en muchas ocasiones presentan una obsolescencia importante y cuentan con un número muy importante de alumnos que son difíciles de ubicar en espacios reducidos.

No obstante se viene observando un esfuerzo importante en inversiones en este apartado. Hay una biblioteca, la Universidad Autónoma de Barcelona, que considera un problema prioritario las fuertes inversiones que será necesario llevar a cabo si se quiere que las Bibliotecas pueden cumplir las funciones encomendadas.

En tercer lugar se sitúan los problemas presupuestarios y cómo afectan las restricciones presupuestarias al servicio de Biblioteca, más en concreto, el no crecimiento de los presupuestos públicos que puede suponer una disminución real de los presupuestos de las Bibliotecas y la necesidad de buscar otras fuentes de financiación: cobro de servicios, subvenciones externas... La escasez presupuestaria agravada por el encarecimiento de los materiales bibliográficos y el aumento de lo costes de personal y funcionamiento es uno de los problemas más graves a lo que se enfrenta la Biblioteca Universitaria. Las Bibliotecas Universitarias disponen en general de presupuesto propio con el que hacer frente a los costes de funcionamiento. Quizá se debe avanzar en la línea del análisis de coste. La gestión del presupuesto propio por el que tanto se ha peleado y por el que se sigue peleando supone una toma de conciencia clara del nivel de madurez que permita ser conscientes de lo que costamos y veamos la forma de hacer "rentable" nuestro servicio.

Curiosamente los recursos bibliográficos, tanto por lo que se refiere a cantidad como a su dispersión quedan situados en el último escalón en su consideración como problema. Son las nuevas Universidades las más preocupadas por la escasez de fondos bibliográficos mientras que son las de tradición las preocupadas por la dispersión y ausencia de control. Se observa una fuerte tendencia centralizadora del fondo bibliográfico, y un mayor control bibliográfico del fondo disperso como consecuencia de la automatización.

La preocupación relativa por el volumen de fondos bibliográficos viene dada por la asunción de que la cooperación es una vía importante 
de acceso al documento y la seguridad de que la información científica y técnica evoluciona en sus soportes y acceso, dejando de tener importancia lo que se posee frente a la que se accede. Se detecta también un cierto abandono de la función conservadora y una tendencia al acceso y difusión de aquello que no se posee. Una de las bibliotecas apuntaba su preocupación acerca de "cómo servimos a los usuarios dada la reconversión tecnológica del medio o soporte de la información científico-técnica».

\section{Conclusiones}

Mayor preocupación por criterios cualitativos que cuantitativos. Síntoma de un cierto grado de madurez. Incluso las nuevas Universidades parten de unos mínimos aceptables.

Temas como calidad de servicio o liderazgo en la Institución, impensables hace 10 años, son en este momento algo prioritario para las Bibliotecas Universitarias.

La automatización parece un tema "superado" en cierto modo, sobre todo en lo que se refiere a elección y puesta en marcha de un sistema de gestión. Siguen existiendo problemas de retroconversión de las Bibliotecas Universitarias de más tradición.

Los problemas de infraestructuras e instalaciones siguen preocupando aunque se están haciendo grandes esfuerzos en las instituciones, un $80 \%$ de las Universidades tienen proyectos de nuevos edificios de Bibliotecas Universitarias en marcha o acaban de finalizarlos.

Los temas presupuestarios preocupan relativamente. Preocupa sobre todo la capacidad para gestionarlos más que su escasez o recorte, algo semejante se puede apuntar en el tema de RR.HH.

Diferencia de interés y prioridades según se trate de Bibliotecas asentadas o bibliotecas de "nueva creación".

Como conclusión general se observa un avance sustancial en estos últimos cinco años y un grado de madurez importante respecto de los temas que interesan. Se destacarían:

- La conciencia de que nos movemos en un entorno tremendamente dinámico.

- La apertura al cambio.

- El interés por no perder el tren y ocupar un lugar central en la Institución que por primera vez toma conciencia de la importancia de los Servicios de Información. 


\section{Pasado, presente y futuro de las bibliotecas...}

\section{Notas}

1 Iglesia Lesteiro, M. ${ }^{a}$ Fernanda y Bernaola Ingunza, M. ${ }^{a}$ Angeles: "Líneas generales para el planeamiento de la biblioteca de la Universidad del País Vasco», en: Boletín de la Anabad, 32(82)3, p. 227-268. Lorenzo MillanA, Carmen: «La biblioteca de la Universidad de Alcalá de Henares”, en: Boletín de la Anabad, 39(89)1, p. 73-89. LlOPART, Pilar, LAMARCA, Dolors: "Les biblioteques de la Universitat de Barcelona des del període de la Universitat Autònoma fins als nostres dies", en: La Biblioteca de la Universitat de Barcelona (Universitat de Barcelona, Barcelona, 1994) p. 60-105.

2 Es un artículo muy general de LASSO DE LA VEGA, Javier que lleva por título "La biblioteca en el plan de reforma de la enseñanza" (Boletín de Anaba, 20: 56 (1970), p. 39-41) publicado el mismo año en ABC.

3 Burrows, Toby: «British University Libraries" (Haworth, New York, 1989).

4 THOMPSON, James: "Redirection in academic library management" (The Library Association, London, 1991).

5 Véanse las "Annual library statistics» del SCONUL y el "Annuaire des bibliothèques universitaires et des grands établissements» de la Direction de la Programmation et du développement universitaire.

6 "La biblioteca en la universidad: informe sobre las bibliotecas universitarias en España, elaborado por el Grupo de Trabajo integrado por bibliotecarios y profesores de Universidad» (Ministerio de Cultura. Dirección General del Libro y Bibliotecas, Madrid, 1985).

7 «Estudio sobre normalización e informatización de las bibliotecas científicas españolas» (Fundesco, Madrid, 1989). No publicado.

8 «Estadística de las bibliotecas universitarias 1994 (datos provisionales): avance de datos" (Consejo de Universidades. Secretaría General, junio Madrid, 1995). No publicado.

9 "Situación de las bibliotecas universitarias (dependientes del M.E.C.)» (Universidad Complutense. Biblioteca, Madrid, 1988).

10 «Les biblioteques de les universitats públiques de Catalunya en els anys 90 = State-funded university libraries in Catalonia in the nineties" (Comissionat per a Universitats i Recerca, Barcelona, 1993).

11 Situación actual y tendencias de futuro de las bibliotecas científicas de la CAM (CSIC, UAH, UAM, UCIII, UCM, UNED, Madrid, [1995]). No publicado.

12 Belmonte Martinez, Isabel: "La biblioteca ante la reforma de la universidad", en: Boletín de la Anabad, 36(86)1-2, p. 167-176.

13 Véase la mención de GONZÁLEZ ANTÓN, Javier a los organigramas "especiales" o "anómalos» en su intervención en el Seminario de la UIMP 'Bibliotecas, ¿Qué servicios y con qué técnicas?' (La Laguna, 1995). No publicado.

14 Con pocas diferencias, ésta es la definición que se incluye en las normas «Bibliotecas universitarias: recomendaciones sobre su reglamentación": Jornadas de trabajo sobre bibliotecas universitarias, "Castillo Magalia", Las Navas del Marqués (Avila), 27-28 de mayo de 1986" (Centro de Coordinación Bibliotecaria, Madrid, 1987) y en las "Recomedaciones para un reglamento marco de bibliotecas universitarias" ([S.l.: Rebiun, 1991). No publicado.

15 Lamarca, Dolors, apuntes del curso sobre bibliotecas universitarias impartido en el Col'legi de Bibliotecaris-Documentalistes de Catalunya (1991). Pueden verse también más datos sobre la centralización/descentralización en el artículo de Moralejo 


\section{Lluís Anglada i de Ferrer y Margarita Taladriz Mas}

Alvarez, Remedios: «La biblioteca universitaria en España, entre tradición y renovación"', en: Boletín de la Anabad, 45(95)1, p. 145-164.

16 GonZÁlez ANTÓN, Javier: op. cit.

17 Abad Hiraldo, Ramón y Moralejo Alvarez, Remedios: «La financiación de las bibliotecas universitarias en España: situación actual y perspectivas», en: 59th IFLA Council and Conference (Barcelona, 1993) Booklet 1, p. 10-15.

18 Informe sobre la situación de las bibliotecas universitarias españolas. (Confencia de directores de bibliotecas universitarias y científicas españolas, mayo de 1995). No publicado.

19 Informe sobre la situación de las bibliotecas universitarias españolas. Op. cit.

20 ORTIZ-REPISO, Virginia; Rfos, Yolanda: «Automated cataloguing and retrospective conversion in the university libraries of Spain*, en: Online \& CDROM review 18(94)3, p. 157-167. KEEFER, Alice y JIMÉNEZ, Miguel: «Library automation in Spain: an overview», en: Program 26(92)3, p. 225-237. Estrvill Rius, Assumpció: «Automation of university libraries in Spain: a status report", en: Telephassa Seminar on Innovative Information Services and Information Handling: proceedings (Tilburg University Library, Tilburg, 1992). Pueden verse, además, CRESPO ARCE, J. B.: "La informatización de las bibliotecas en la Comunidad Europea: estudio comparativo" (Sedic, Madrid, 1993) y Moscoso, Purificación; Rios Garcia, Yolanda: «Estado actual de la aplicación de las nuevas tecnologías de la información en las bibliotecas y su impacto sobre el funcionamiento bibliotecario : puesta al día : LIB-2/13-update (Spain)» ([S.1.]: Fesabid, 1992).

21 Puede verse un análisis del distinto grado de implementación de módulos de los sistemas automatizados en la ponencia de Estrvill RIUS, Assumpció citada anteriormente.

22 Puede verse una descripción completa del proceso de la UPC en el artículo de ANGladA, Lluís; PÉrez, Adoració y Puertas, Miquel: "La conversión retrospectiva en la Universitat Politècnica de Catalunya», en: Boletín de la Anabad 42(92)2, p. 76-94.

23 Informe sobre la situación de las bibliotecas universitarias españolas. Op. cit.

24 Pueden verse las sugerentes reflexiones de los muy reputados CRAWFORD, Walt y Gorman, Michael en: «Future libraries: dreams, madness \& reality» (ALA, Chicago, 1995).

25 GonZÁlez ANTÓN, Javier op. cit., es de la misma opinión aunque no aporta datos adicionales.

26 «Enquesta sobre l'ús i la satisfacción de les biblioteques de la UPC per part dels estudiants: curs 1994-95» (Biblioteques de la UPC, Barcelona, 1996). No publicado.

27 Pueden verse estos temas tratados con más profundidad en el artículo de ANGLADA I DE FERRER, Lluís M. a: "Cooperació bibliotecaria a Espanya», en: Item 16(95), p. 51-67.

28 AmaT, Lluïsa: «La Coordinadora de Documentació Biomèdica» (1995). No publicado.

29 Rodrfguez Alvarez, Ramón et al.: «La red universitaria española DOBIS/LIBIS», en: Documat 94, p. 253-259.

30 HERMS Information Research: total quality management: the information business; key Asone 93. University of Hertfordshire, 1993.

31 Así se recoge expresamente en prácticamente todos los Estatutos de las diferentes Universidades. 